DOI: https://doi.org/10.46296/yc.v5i9ucedespsoct.0127

\title{
POTENCIALIDADES PARA LA PRODUCCIÓN DE LECHE FERMENTADA SIMBIÓTICA DE CABRA EN CAMAGÜEY
}

\section{POTENTIALITIES FOR THE PRODUCTION OF SYMBIOTIC FERMENTED GOAT MILK IN CAMAGÜEY}

\author{
Mustelier-Casola Daimara ${ }^{1}$; Álvarez-Serrano Isaris María ${ }^{2}$; \\ Nápoles-Abreu Osvaldo ${ }^{3}$; Crespo-Zafra Lourdes Mariana ${ }^{4}$
}

${ }^{1}$ Profesor del departamento de Ciencia y Tecnología de los Alimentos de la Universidad de Camagüey "Ignacio Agramonte Loynaz". Camagüey, Cuba. Correo: daimara.mustelier@reduc.edu.cu. ORCID ID: https://orcid.org/00000001-5284-50464

${ }^{2}$ Profesor del departamento de Ciencia y Tecnología de los Alimentos de la Universidad de Camagüey "Ignacio Agramonte Loynaz". Camagüey, Cuba. Correo: isaris.alvarez@reduc.edu.cu. ORCID ID: https://orcid.org/00000001-7772-6262

${ }^{3}$ Profesor del departamento de Ciencia y Tecnología de los Alimentos de la Universidad de Camagüey "Ignacio Agramonte Loynaz". Camagüey, Cuba. Correo: osvaldo.napoles@reduc.edu.cu. ORCID ID: https://orcid.org/0000-0003-0658-4257

${ }^{4}$ Profesor del departamento de Ciencia y Tecnología de los Alimentos de la Universidad de Camagüey "Ignacio Agramonte Loynaz". Camagüey, Cuba. Correo: lourdes.crespo@reduc.edu.cu. ORCID ID: https://orcid.org/0000-0002-4799-3447

\section{Resumen}

Actualmente la producción caprina en Cuba se presenta como una interesante alternativa para la obtención de productos de alto valor biológico. Diversos autores indican cómo las proteínas de la leche de cabra resultan frente a la de vaca, menos alergénicas y de mayor absorción. Estudio reciente, realizado en Camagüey confirmó la demanda creciente en la población tanto de leche de cabra como de los productos derivados. Entre los productos de leche caprina y en el campo de los alimentos funcionales han ganado lugar las leches fermentadas simbióticas, las cuales son obtenidas a partir de la combinación de microorganismos probióticos y suplementos prebióticos. Este trabajo tiene como objetivo valorar las potencialidades para la producción de leche fermentada simbiótica de cabra en la provincia de Camagüey, para personas con necesidades nutricionales especiales. El diseño de esta investigación fue no experimental, transaccional y correlacional. Hasta la actualidad se han realizado en Cuba diversos estudios relacionados con la producción de leches fermentadas a partir de leche de vaca, sin embargo, no se ha aprovechado lo suficiente la leche caprina para la elaboración de leches fermentadas simbióticas. La provincia de Camagüey tiene potencialidades en cuanto a la producción de materias primas, tecnología y recursos humanos para llevar a cabo un proyecto productivo de leche fermentada simbiótica a partir de leche de cabra. Este producto podría atenuar la poca disponibilidad en el mercado de alimentos para personas con necesidades nutricionales especiales en el territorio.

Palabras claves: Leche de cabra, leches fermentadas, fructooligosacáridos y probióticos.

\footnotetext{
Abstract

Currently, goat production in Cuba is presented as an interesting alternative to obtain products of high biological value. Various authors indicate how the proteins in goat's milk are less allergenic and more absorbable compared to cow's milk. A recent study, carried out in Camagüey confirmed the growing demand in the population for both goat's milk and derived products. Among goat milk

Información del manuscrito:

Fecha de recepción: 26 de julio de 2021.

Fecha de aceptación: 29 de septiembre de 2021.

Fecha de publicación: 11 de octubre de 2021.
} 
products and in the field of functional foods, symbiotic fermented milks have gained place, which are obtained from the combination of probiotic microorganisms and prebiotic supplements. This work aimed to assess the potential for the production of symbiotic fermented goat milk in Camagüey province, for people with special nutritional needs. The design of this research was non-experimental, transactional, and correlational. Until now, various studies related to the production of fermented milk from cow's milk have been carried out in Cuba, however, goat milk has not been used enough as a raw material for the production of functional foods. Camagüey province has the raw materials, technological capacity and human potential necessary for the production of symbiotic fermented milk from goat's milk. This product could mitigate the limited availability in the market in this province of food for people with special nutritional needs.

Keywords: Goat milk, fermented milk, fructooligosaccharides and probiotics.

\section{Introducción}

Actualmente la leche caprina y sus derivados se colocan en el mercado con un valor nutricional importante, es considerada un alimento funcional natural debido a que reporta beneficios para la salud y reduce el riesgo de enfermedades. Diversos autores indican cómo las proteínas de la leche de cabra resultan frente a la de vaca, menos alergénicas. Señalan cómo el aprovechamiento de la primera resulta mejor, en razón de que forma en el estómago un coágulo más pequeño, blando y fragmentable, circunstancias que favorecen la acción de las proteasas digestivas (Figueredo, 2020).

Cuando se habla de necesidades nutricionales especiales, para esta investigación, se identifica a un grupo de individuos afectados por enfermedades gastroenterológicas, reacciones alimentarias adversas 0 por otros padecimientos asociados a la absorción y metabolismo de nutrientes. El término reacción alimentaria adversa se refiere a cualquier síntoma presente en forma constante y repetida después de la ingestión de un determinado alimento, reacción que puede deberse a una alergia o intolerancia (Ignorosa, Loredo, Cervantes, Zárate, Montijo, Toro \& Ramírez, 2017).

Entre los productos de leche caprina $y$ en el campo de los alimentos funcionales han ganado lugar las leches fermentadas simbióticas, las cuales son obtenidas a partir de la combinación de microorganismos probióticos y suplementos prebióticos. Entre los beneficios más conocidos de los productos simbióticos se pueden encontrar: efecto potenciador sobre el sistema 
inmune, actividad detoxificante, anticancerígena, acción preventiva contra

enfermedades

cardiovasculares, amplio espectro de inhibición sobre invasores patógenos, entre otros (Agirre, 2020).

La leche fermentada probiótica es un alimento que se define como aquel producto que contiene microorganismos viables que ingeridos en cantidades adecuadas ejercen un efecto beneficioso para la salud del consumidor, contiene una mezcla de cultivos lácteos que se incorporan a la leche con el objetivo de obtener un producto con características específicas (Vénica, Bergamini, Wolf \& Perotti, 2020).

Entre los prebióticos que más se utilizan en las leches fermentadas simbióticas se encuentran los fructooligosacáridos. Estos carbohidratos popularmente llamados FOS, presentan dos propiedades fisiológicas características: su no-digestibilidad y su utilización selectiva por el microbiota intestinal beneficiosa. El consumo per cápita de FOS en los Estados Unidos está estimado entre 1-4 g/día y en Europa entre 3-11 g/ día. Otros autores plantean el consumo de 0,7 g/ día, en el caso de los niños, y alrededor de 1,5 g/día para adultos (Játiva, Manterola, Macias \& Narváez, 2021).

En Cuba, desde el 2008, la seguridad alimentaria ha sido declarada por la máxima dirección del gobierno como una prioridad nacional. Ante el compromiso de impulsar y diversificar la producción de alimentos para el consumo humano en el país, se promueve el desarrollo de las producciones caprinas, a través del Lineamiento 164 de la Política Económica y Social del Partido y la Revolución aprobada en el 7 mo Congreso del Partido y la Asamblea Nacional en el 2016, que manifiesta la voluntad de ..." desarrollar el ganado menor en las regiones del país con condiciones favorables para ello".

En este sentido, en la provincia de Camagüey, recientemente se han gestado iniciativas locales con el propósito de aprovechar el potencial productivo existente para lidiar con los desafíos que se presentan ante la aspiración de alcanzar la satisfacción de las necesidades locales. Muestra de ello lo constituye el proyecto territorial "La gestión de innovación en el encadenamiento 
productivo de la agroindustria azucarera en el municipio Florida". En este proyecto los investigadores del departamento de Ciencia y Tecnología de los Alimentos de la Universidad de Camagüey persiguen implementar innovaciones que permitan obtener derivados lácteos caprinos con elevado valor agregado y que contribuyan a sustituir importaciones, incentivar exportaciones e inducir al cierre de ciclos productivos en función de la seguridad alimentaria.

Como un valioso antecedente, en 2017, se realizó un estudio de demanda para diferentes derivados de leche de cabra en la provincia de Camagüey, en el marco del proyecto "Aplicación de tecnologías que contribuyan al incremento productivo de la leche caprina y al desarrollo de producciones secundarias en la cadena agro-industrial". Para ello se aplicaron instrumentos específicos a directivos del Ministerio de la Agricultura, a técnicos y directivos de producciones lácteas, fueron encuestados pacientes consumidores de leche de cabra por prescripción facultativa, ancianos escogidos al azar y consumidores de leche de vaca. Este estudio arrojó como resultado que existe una demanda creciente en la población tanto de leche de cabra como de los productos derivados (Matos, Crespo \& Bidot, 2017).

Se obtuvo además que en dicho año estaban reportados 1520 niños menores de 6 años con trastornos nutricionales asociados al consumo de leche, de los cuales el 86,97\% correspondía a intolerantes a la lactosa y el 13,03\% restante presentaba alergia a las proteínas de la leche de vaca.

Por otro lado, como sustituto para los alérgicos existía la fórmula Prolacsin, un alimento completo, libre de proteína láctea y lactosa para niños intolerantes, elaborado por el Instituto de Investigaciones para la Industria Alimentaria, compuesto por grasa vegetal, aislado de proteína de soya, azúcar refino, grasa láctea, maltodextrina, vainilla etílica y además enriquecido con vitaminas y minerales. Sin embargo, este alimento no era capaz de suplir el $50 \%$ de las necesidades de la población con estos padecimientos. Además, se realizaban asignaciones normadas de yogur natural, con lo que un $27 \%$ de los pacientes no apreciaba mejoría de los síntomas 
(2018, Informe técnico de culminación de proyecto).

Atendiendo a las necesidades de consumo de personas con necesidades nutricionales especiales se propuso la elaboración de un producto lácteo de origen caprino en el territorio, que a la vez supliera los requerimientos nutricionales de estos pacientes y aprovechara las características beneficiosas de la leche de cabra. De ahí que, este trabajo tiene como objetivo valorar las potencialidades para la producción de leche fermentada simbiótica de cabra en Camagüey.

\section{Metodología (Materiales y métodos)}

El presente estudio, se realizó mediante una revisión bibliográfica utilizando fuentes de información primarias y secundarias, lo cual permitió la construcción del marco de referencia teórico y metodológico. El diseño de la investigación fue no experimental, transaccional $y$ correlacional. El estudio sostuvo además como método a la observación no participativa para la recopilación de información, y como técnica al análisis documental para la obtención de datos reales y el fortalecimiento de los resultados. Para la valoración realizada sobre las potencialidades para la producción de leche fermentada simbiótica de cabra en Camagüey, se tuvieron en cuenta los indicadores siguientes: experiencias de los últimos seis años en la producción de leches fermentadas probióticas en Cuba, disponibilidad de materias primas, infraestructura industrial y potencial humano.

\section{Resultados}

Experiencias en la producción de leches fermentadas probióticas en Cuba

En los últimos seis años se han realizado en Cuba varios estudios experimentales sobre leches fermentadas probióticas (Tabla 1). Estas experiencias podrían ser útiles para el desarrollo de un diseño experimental para la elaboración de una leche fermentada simbiótica de cabra. En casi todos estos estudios se ha utilizado como materia prima fundamental la leche de vaca, ya sea entera o descremada. 
Tabla 1. Estudios experimentales sobre leches fermentadas probióticas realizados en Cuba en el periodo de 2016-2021.

\begin{tabular}{|c|c|c|}
\hline Estudio & Materias Primas & Autor \\
\hline $\begin{array}{l}\text { Desarrollo de una leche } \\
\text { fermentada de cabra con } \\
\text { cultivos probióticos }\end{array}$ & $\begin{array}{l}\text { Leche de cabra } \\
\text { Lactobacillus casei } \\
\text { Lactobacillus acidophillus } \\
\text { Lactobacillus bulgaricus } \\
\text { Streptococcus thermophillus }\end{array}$ & $\begin{array}{c}\text { (Hernández, Torres, } \\
\text { Duarte y Villacis, 2016) }\end{array}$ \\
\hline $\begin{array}{l}\text { Leche fermentada simbiótica } \\
\text { con sirope prebiótico cubano }\end{array}$ & $\begin{array}{l}\text { Leche de vaca } \\
\text { Lactobacillus acidophillus } \\
\text { FOS }\end{array}$ & $\begin{array}{l}\text { (Rodríguez, Rodríguez, } \\
\text { Trujillo, Pérez, León, } \\
\text { Hernández y Santos, } \\
\text { 2017) }\end{array}$ \\
\hline $\begin{array}{l}\text { Desarrollo de una leche } \\
\text { fermentada a partir de leche de } \\
\text { búfala y cultivo de Lactobacillus } \\
\text { acidophilus }\end{array}$ & $\begin{array}{l}\text { Leche de búfala } \\
\text { Lactobacillus acidophillus } \\
\text { Emulsión de naranja }\end{array}$ & $\begin{array}{l}\text { (Íñiguez, Rodríguez, } \\
\text { Hernández, Martínez y } \\
\text { Nieto,2018) }\end{array}$ \\
\hline $\begin{array}{l}\text { Evaluación de la estabilidad de } \\
\text { prebióticos durante el proceso } \\
\text { de elaboración y conservación } \\
\text { de una leche fermentada } \\
\text { simbiótica }\end{array}$ & $\begin{array}{l}\text { Leche de vaca } \\
\text { Cultivos Bioyogur } \\
\text { FOS }\end{array}$ & $\begin{array}{l}\text { (Rodríguez, Pérez, } \\
\text { Martínez, Fernández, } \\
\text { Pavón y Jiménez, 2019) }\end{array}$ \\
\hline $\begin{array}{l}\text { Empleo de harina de arroz en } \\
\text { una leche fermentada probiótica }\end{array}$ & $\begin{array}{l}\text { Leche de vaca } \\
\text { Cultivos Bioyogur } \\
\text { Harina de arroz }\end{array}$ & $\begin{array}{l}\text { (Valdés, Guzmán, } \\
\text { Iñíguez, Rodríguez, y } \\
\text { Martínez, 2020) }\end{array}$ \\
\hline $\begin{array}{l}\text { Empleo de pulpas de acerola y } \\
\text { guayaba en una leche } \\
\text { fermentada probiótica }\end{array}$ & $\begin{array}{l}\text { Leche de vaca } \\
\text { Cultivos Bioyogur } \\
\text { Pulpa de acerola y guayaba }\end{array}$ & $\begin{array}{l}\text { (Valdés, M’Moumba, } \\
\text { Iglesias, Castro, } \\
\text { Bejerano, Rodríguez y } \\
\text { Guzmán, 2020) }\end{array}$ \\
\hline $\begin{array}{l}\text { Caracterización de una leche } \\
\text { fermentada probiótica } \\
\text { endulzada con sirope }\end{array}$ & $\begin{array}{l}\text { Leche de vaca } \\
\text { Cultivos Bioyogur } \\
\text { Sirope } 67 \text { Bx: Agua, azúcar, } \\
\text { sabor fresa, color rojo. }\end{array}$ & $\begin{array}{l}\text { (Valdés, Castro, } \\
\text { Bejerano, Martínez y } \\
\text { Guzmán, 2020) }\end{array}$ \\
\hline $\begin{array}{l}\text { Empleo de pulpas de acerola y } \\
\text { mango en una leche } \\
\text { fermentada probiótica }\end{array}$ & $\begin{array}{l}\text { Leche de vaca } \\
\text { Cultivos Bioyogur } \\
\text { Pulpa de acerola y mango }\end{array}$ & $\begin{array}{l}\text { (Valdés, M’Moumba, } \\
\text { Iglesias, Castro, } \\
\text { Bejerano, Rodríguez y } \\
\text { Guzmán, 2021) }\end{array}$ \\
\hline
\end{tabular}

Fuente: Elaboración de los autores. 
El Lactobacillus acidophilus es el cultivo con características probióticas más utilizado en el país, ya sea asociado con otras cepas entre las que resalta el cultivo láctico Bioyogur (cocultivo de Lactobacillus acidophilus y Streptococcus thermophilus) procedente del Banco de Cepas del Instituto de Investigaciones para la Industria Alimenticia (IIIA) o solo, dando como resultado una leche comúnmente conocida como leche acidófila.

Específicamente a L. acidophilus le son atribuidas las propiedades de mantener el estreñimiento y la diarrea bajo control, contrarrestar la intolerancia a la lactosa por su asociación con lactasa, la reducción de los gases internos, el control del colesterol mediante una absorción normal de grasas en la dieta, así como la prevención de la contaminación por enfermedades infecciosas intestinales (Íñiguez et al. 2018).

Las bacterias probióticas deben consumirse a concentraciones celulares mayores de $107 \mathrm{ufc} / \mathrm{mL}$ para poder ejercer su efecto probiótico. Sin embargo, el crecimiento de estas bacterias durante el período de conservación de un producto, puede afectarse por diferentes causas. Es por ello que se ha estudiado el efecto de la incorporación de los FOS en las leches cuando son fermentadas con microorganismos probióticos. Entre los prebióticos más utilizados en alimentos se encuentran las inulinas y fructooligosacáridos. Los resultados fueron satisfactorios pues la fuerza del coágulo y viabilidad del probiótico durante la conservación resultaron mayores en la leche fermentada con sirope FOS (Rodríguez et al. 2017).

Por otra parte, resultó necesario conocer si durante la conservación el desarrollo de los microorganismos presentes en la leche fermentada 0 las condiciones ácidas y de actividad de agua de la matriz podrían conducir a alguna disminución en la concentración de los fructooligosacáridos. Las condiciones de temperatura a las que se expone el alimento también constituyen inconvenientes para el mantenimiento de la estructura de los fructooligosacáridos. Por estas razones se evaluó la concentración del fructooligosacárido antes y después de la pasteurización y durante la conservación de una 
leche fermentada simbiótica. Como resultado se mantuvieron invariables las fracciones de prebióticos de la leche fermentada simbiótica después de la pasteurización, la inoculación y durante toda la conservación (Rodríguez et al. 2019).

En todas estas investigaciones revisadas, las leches fermentadas desarrolladas

presentaron adecuadas características composicionales y una alta valoración sensorial. Los indicadores microbiológicos cumplieron las especificaciones establecidas, avalando la adecuada calidad higiénico sanitaria y la viabilidad celular del probiótico. Estos aspectos demuestran el destino de estos productos para fines dieto terapéuticos.

\section{Disponibilidad de materias primas}

Figura 1. Aporte a la producción de leche de cabra total por territorios (\%) en el periodo 2012-2017.

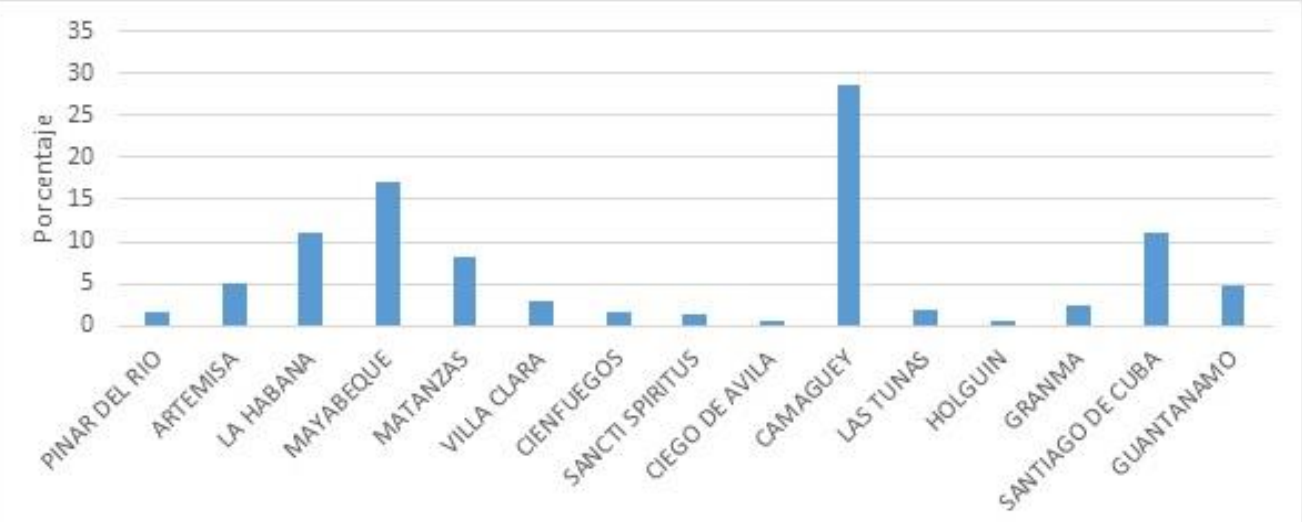

Fuente: Informe técnico de culminación del proyecto (2018).

\section{Leche de cabra}

Según el Informe técnico de culminación del proyecto "Aplicación de tecnologías que contribuyan al incremento productivo de la leche caprina y al desarrollo de producciones secundarias en la cadena agroindustrial 2014-2018" (2018) en el periodo 2012-2017 Camagüey producía los mayores volúmenes de leche caprina del país, como se muestra en la figura 1.

En 2017, como parte de las acciones de los programas para el desarrollo de la ganadería ovino-caprina, auspiciados por el Grupo de Flora y Fauna, en los municipios de Sibanicú y Jimaguayú, se potenció el mejoramiento de los rebaños, además de fortalecer la base alimentaria de los animales (Radio Cadena Agramonte, 2017). 
En 2019, la provincia Camagüey contaba con alrededor de 220 productores dedicados a la cría de cabras, quienes se propusieron en ese año superar la entrega de 800 mil litros de leche a la industria láctea. En el empeño por contribuir al desarrollo de esa masa de animales, la Asociación Nacional de Agricultores Pequeños (ANAP) también socializó conocimientos y fomentó la implementación de proyectos locales (Radio Cadena Agramonte, 2019).

\section{Disponibilidad de prebióticos}

En Cuba, se desarrolló por primera vez la producción de un sirope FOS mediante la fermentación de una melaza de caña por la levadura Pichia pastoris. Este producto se comercializó con el nombre de "ALIDUX", constituyendo el primer prebiótico de producción nacional. Se ha demostrado que el país puede producir la enzima de origen vegetal que transforma la sacarosa en el referido producto. En 2018, se fabricaron 6.28 toneladas, gracias a la imbricación entre el Centro de Ingeniería Genética y Biotecnología (CIGB), el Instituto Cubano de Investigaciones de los Derivados de la Caña de Azúcar (ICIDCA) y la Planta de Sorbitol UEB "Ignacio Agramonte" ubicada en el municipio de Florida, perteneciente a la Empresa Azucarera Camagüey (Rodríguez et al. 2019).

Actualmente, se dispone de un sirope de FOS $55 \%$ de pureza, concentrado a $75^{\circ} \mathrm{Bx}$. Este producto que se comercializa con el nombre de "KestoMix" ha sido sometido a análisis y evaluación del registro nacional de acuerdo a lo establecido en la legislación vigente, encontrándose apto para el uso y consumo humano, según la Licencia Sanitaria No. PN-16233/18. El sirope FOS $55 \%$, tiene características y parámetros físico-químicos similares a productos de igual grado de purezas existentes en el mercado, pero con una composición en 1 kestosa superior en más de un $10 \%$.

En su producción se empleó como sustrato de la síntesis un azúcar con las siguientes características: Pol: 99,80Z, Humedad: 0,062 \% m/m, Cenizas: $0,032 \% \mathrm{~m} / \mathrm{m}$, Color ICUMSA: $70 \mathrm{UCl}$, Partículas ferromag: $0 \mathrm{mg} / \mathrm{kg}$ (Fonte, 2019).

El producto final tiene la composición que se muestra en la tabla 2 y que 
indica el productor (en la etiqueta del envase).

Tabla 2. Componentes del Sirope FOS concentrado a 75ํㅡ. Envase $240 \mathrm{~g}$.

\begin{tabular}{lc}
\hline Ingredientes & $\begin{array}{r}\% \\
\text { (masa/masa) }\end{array}$ \\
\hline 1- Kestosa & $47-54$ \\
Nistosa & $3-9$ \\
Fructosa & Menor que 4 \\
Glucosa & $20-25$ \\
Sacarosa & $15-22$ \\
\hline
\end{tabular}

Fuente: Elaboración de los autores.

El productor recomienda un porción aporta 13,4 kcal, como se consumo de no más de tres observa en la tabla 3 (tomado de la porciones de $5 \mathrm{ml}$ por día, cada etiqueta del envase).

Tabla 3. Información nutricional por porción de $5 \mathrm{ml}$

\begin{tabular}{|l|l|}
\hline Calorías & $13,4 \mathrm{kcal}$ \\
\hline Grasa & $0 \mathrm{~g}$ \\
\hline Proteínas & $0 \mathrm{~g}$ \\
\hline Carbohidratos totales & $5,2 \mathrm{~g}$ \\
\hline Fibra dietética & $2,9-3,1 \mathrm{~g}$ \\
\hline Azúcares & $2,1-2,3 \mathrm{~g}$ \\
\hline
\end{tabular}

Fuente: Elaboración de los autores.

\section{Infraestructura industrial}

Las principales líneas de producción para el consumo directo de la empresa Productos Lácteos Camagüey, están constituidas por: leche fluida, yogurt de leche y de soya, helados, mantequilla, quesos de diferentes surtidos $y$ otros derivados de la leche y la soya. Esta empresa contó en 2017 con mejorías en sus instalaciones y aumento de capacidad. Emprendió la sustitución de muchos equipos y sistemas, sobre todo tuberías, que presentaban un elevado nivel de deterioro y provocaban constantes paradas del proceso. Las reparaciones se hicieron en la fábrica de helados Coppelia, en esta 
ciudad, en las pasteurizadoras de Nuevitas, Florida y Santa Cruz del Sur, y en las queseras La Vaquita, Taíno y Sibanicú (Juventud Rebelde, 2017).

Entre sus unidades empresariales de base la empresa láctea de Camagüey cuenta con la Planta Pasteurizadora, su principal industria, la cual continúa el proceso gradual de mejoramiento tecnológico a fin de potenciar la calidad y seguridad sanitaria de los alimentos que elabora. Además, existe la Fábrica de Leche en Polvo, la cual posee un laboratorio de microbiología climatizado, con cinco áreas de riesgo, que funciona según el Principio de Caja en Caja, y un Laboratorio de físico - químicos en el cual el $80 \%$ de los equipos están diseñados especialmente para productos lácteos, esta industria reúne las condiciones requeridas para realizar los análisis pertinentes a la leche fermentada simbiótica de cabra.

Por otro lado, para realizar en un primer momento los estudios experimentales necesarios, la Universidad de Camagüey posee una Planta Piloto que actualmente cuenta con líneas especializadas, entre ellas: cervezas y maltas, vinos, rones y aguardientes, conservas de vegetales, derivados lácteos, y cereales; y posee un laboratorio de análisis físico-químicos.

\section{Potencial humano}

En Cuba, la máxima dirección del gobierno ha insistido en la importancia de potenciar lo local como espacio estratégico para la gestión del desarrollo. Para ello, se plantea que se requieren reconocer las capacidades locales para aprovechar las oportunidades y lidiar con las dificultades mediante el despliegue de su potencial. Se promueve la articulación de lo territorial con lo nacional en ambos sentidos para favorecer la sinergia de su economía, sociedad, las personas, empresas e instituciones, en el diseño y realización de sus proyectos colectivos e individuales.

En la provincia de Camagüey coexisten un conjunto de actores, individuos $\mathrm{y} / \mathrm{u}$ organizaciones, que intervienen en el proceso de agregación de valor de la cadena agroalimentaria de leche de cabra y sus derivados. Los servicios que brindan éstos pueden ser de transformación o comercialización 
del producto, logísticos, financieros, de capacitación o asistencia técnica, así como de regulación de determinados procesos. En este sentido, se destaca la Empresa de Productos Lácteos Camagüey que alberga una amplia experiencia en la producción de leches fermentadas, por ser esta una provincia históricamente productora de leche y derivados. En sus industrias, constan con personal técnico calificado para asumir las producciones de este producto. No obstante, la capacitación constante constituye un pilar fundamental, tanto para técnicos industriales como agropecuarios.

Según Gispert, Pedraza, Vázquez y Bidot (2020) en Camagüey, la mayoría de los caprinocultores son menores de 50 años y tienen un nivel escolar secundario, lo cual es adecuado para asimilar un proceso de capacitación sobre el manejo del ganado caprino lechero. De acuerdo con Delgado, Borroto, Barreto, Rodríguez y Peña (2017) la transferencia de conocimientos a productores individuales contribuye al mejoramiento del manejo sanitario y de los indicadores bioproductivos en fincas caprinas.
Los principales referentes del servicio de capacitación del sector agropecuario son las escuelas del sistema de capacitación del Ministerio de la Agricultura (MINAG), universidades locales y centros de investigación del territorio. La Asociación Cubana de Producción Animal (ACPA) y la Asociación Cubana de Técnicos Agrícolas y Forestales (AFTAF) juegan un rol importante en la formación y capacitación. ACPA lleva a cabo la capacitación integral a los productores de todas las formas productivas mediante conferencias, entrenamientos, análisis de artículos científicos, capacitación en la finca de productores, entrega de revistas técnicas folletos y plegables. Por su parte, la ACTAF representa a técnicos y profesionales del sector agropecuario y forestal en todos los espacios donde se promueve el desarrollo sostenible sobre bases agroecológicas. Entre sus misiones están la construcción de espacios de intercambio y diálogo con equidad entre el personal técnico, agropecuario y forestal, para la promoción de formas de producción sostenibles sobre bases agrosostenibles, el rescate del 
conocimiento y las tradiciones del patrimonio sociocultural, entre otras.

Como señalan Díaz-Canel y Fernández (2020), las universidades cubanas constituyen un actor relevante en los procesos de producción, difusión y uso de los conocimientos que demanda la sociedad local, reconociéndose como un aliado clave de los gobiernos en la conducción estratégica del desarrollo local. Una muestra de este aporte es el manual confeccionado por profesores de la Universidad de Camagüey y la Universidad de Ciencias Médicas de Ciego de Ávila, que tiene el propósito de transferir conocimientos a caprinocultores, referido al uso de microorganismos autóctonos multipropósito (MAM). Este manual es de gran utilidad tanto para mejorar los parámetros productivos en los rebaños, al estimular la producción de leche, como para prevenir la presencia de enfermedades infecciosas, evitar los malos olores, tratar el agua de consumo y otras aplicaciones de interés.

La Universidad de Camagüey posee, además, el Departamento de Ciencia y Tecnología de los Alimentos, con personal preparado para brindar la capacitación necesaria en las tecnologías de producción, en inocuidad alimentaria, seguridad y en evaluación de la calidad de leches fermentadas. En el año 2017, se realizaron varios estudios investigativos sobre producciones de leche en polvo, queso y yogurt de leche de cabra, definiéndose las tecnologías para su producción y se trabajó en la capacitación de productores de varias provincias del país (Matos, Crespo \& Bidot, 2017).

Actualmente, se continúa trabajando en investigaciones acerca de los derivados lácteos caprinos a partir de las experiencias previas lo cual puede contribuir al mejoramiento del conocimiento y en consecuencia al desarrollo de producciones eficientes.

\section{Conclusiones}

En Cuba se han realizado diversos estudios relacionados con la producción de leches fermentadas a partir de leche de vaca, sin embargo, no se ha aprovechado lo suficiente la leche caprina como una materia prima para la elaboración de leches fermentadas simbióticas. $\mathrm{La}$ provincia de Camagüey tiene 
potencialidades en cuanto a la producción de materias primas, tecnología y recursos humanos para llevar a cabo un proyecto productivo de leche fermentada simbiótica de leche de cabra. Este producto podría atenuar la escasa disponibilidad de alimentos en el mercado para personas con necesidades nutricionales especiales en el territorio.

\section{Bibliografía}

Agirre (2020). Actualización sobre probióticos, prebióticos y simbióticos. El farmacéutico, 594, 32-38. Recuperado de: https://www.elfarmaceutico.e s/tendencias/teinteresa/actualizacion-sobreprobioticos-prebioticos-ysimbioticos 111317 102.html

Delgado, R., Borroto, A., Barreto, G., Rodríguez, H., \& Peña, P. (2017). Contribución de la transferencia de conocimientos al manejo sanitario en fincas caprinas. Pastos y Forrajes, 40(3), 216221 , e- ISSN 2078-8452. Recuperado de: http://ref.scielo.org/4bgpwv

Díaz-Canel Bermúdez, Miguel, \& Fernández González, Aurora. (2020). Gestión de gobierno, educación superior, ciencia, innovación y desarrollo local.
Retos de la Dirección, 14(2), 5-32. Epub 02 de diciembre de 2020. Recuperado en 18 de julio de 2021, de https://revistas.reduc.edu.cu/i ndex.php/retos/article/view/35 71

Figueredo, G. (2020). La cabra para los millenials. Un diagnóstico frente a los conocimientos, actitudes y prácticas de la leche de cabra y sus productos derivados. (Tesis de Maestría, Universidad EAFIT, Medellín, Colombia). Recuperado de: https://repository.eafit.edu.co/ handle/10784/17478

Fonte, A. (2019). Estudio del escalado de la producción de Fructooligosacáridos (FOS) a nivel industrial en la planta de Sorbitol. Recuperado de: http://www.researchgate.net/ publication/330856772

Gispert, A., Pedraza, R., Vázquez, R., \& Bidot, A. (2020). Características generales de sistemas familiares de producción caprina del municipio Camagüey, Cuba. Revista Porducción Animal, 31(3), 25-30, ISSN 22247920. Camagüey, Cuba: Universidad de Camagüey "Ignacio Agramonte Loynaz", Facultad de Ciencias Agropecuarias, Centro de Estudios de Producción Animal (CEDEPA). Recuperado de: 
https://revistas.reduc.edu.cu/i ndex.php/rpa/article/view/e32 65

Hernández, A., Torres, A., Duarte, C., \& Villacis, D. (2016). Desarrollo de una leche fermentada de cabra con cultivos probióticos. Tecnología Química, 36(3), 321-335, e-ISSN: 2224-6185. Universidad de Oriente Santiago de Cuba, Cuba. Recuperado de: http://scielo.sld.cu/scielo.php ?script=sci_arttext\&pid=S222 4-61852016000300002

Ignorosa, K., Loredo, A., Cervantes, R., Zárate, F., Montijo, E., Toro, E., ...\& Ramírez, J. (2017). Absorción intestinal deficiente de lactosa; actualidades en pediatría. Alergias, asma e inmunologías pediátricas, 26(1), 49-55. Recuperado de: https://www.medigraphic.com /cgi-

bin/new/resumen.cgi?IDARTI CULO=74335

Informe técnico de culminación del proyecto "Aplicación de tecnologías que contribuyan al incremento productivo de la leche caprina y al desarrollo de producciones secundarias en la cadena agroindustrial 2014-2018". (2018). Programa Nacional de Alimento Humano, La Habana, Cuba.
Íñiguez, C., Rodríguez, T., Hernández, O., Martínez, I., \& Nieto, M. (2018). Desarrollo de una leche fermentada a partir de leche de búfala y cultivos de Lactobacillus acidophilus. Ciencia y Tecnología de Alimentos, 28(2), 1-6. Recuperado de: https://www.revcitecal.iiia.edu .cu/revista/index.php/RCTA/a rticle/view/53

Játiva, E., Manterola, C., Macias, R., \& Narváez, D. (2021). Probióticos y Prebióticos. Rol en la Terapéutica de la Enfermedad Diarreica Aguda Infantil. Int. J. Morphol, 39(1), 294-301. Recuperado de: https://www.scielo.cl/scielo.ph p?script=sci_arttext\&pid=S07 17-95022021000100294

Juventud Rebelde. (2017). Industria láctea de Camagüey sigue mejoramiento tecnológico. Recuperado de: www.juventudrebelde.cu

Matos, L., Crespo, L., \& Bidot, A. (2017). Soberanía Alimentaria y Desarrollo Sostenible: Una Contribución del Licenciado en Ciencias Alimentarias. Ciencias de la Ingeniería y Aplicadas, 2(1), 57-67, ISSN: 2602-8255. Recuperado de: http://investigacion.utc.edu.ec /revistasutc/index.php/ciya/art icle/view/125

Radio Cadena Agramonte. (2017). Se recuperan en Camagüey programas de la ganadería 
ovino-caprina. Recuperado de:

http://www.cadenagramonte.c $\mathrm{u}$

Radio Cadena Agramonte. (2019). Estimulan en Camagüey el incremento de la cría de cabras. Recuperado de: www.cadenagramonte .cu

Rodríguez, O., Pérez, E., Martínez, D., Fernández, J., Pavón, M., \& Jiménez, L. (2019). Evaluación de la estabilidad de prebióticos durante el proceso de elaboración y conservación de una leche fermentada simbiótica. Ciencia y Tecnología de los Alimentos, 29(2), 48-53. Recuperado de: https://www.revcitecal.iiia.edu .cu/revista/index.php/RCTA/a rticle/view/25

Rodríguez, O., Rodríguez, J., Trujillo, L., Pérez, E., León, Y., Hernández, O., ... \& Santos, B. (2017). Leche fermentada simbiótica con sirope prebiótico cubano. Ciencia y Tecnología de los Alimentos, 27(3), 21-27. Recuperado de: https://www.revcitecal.iiia.edu .cu/revista/index.php/RCTA/a rticle/view/112

Valdés, M., Castro, Y., Bejerano, D., Martínez, I., \& Guzmán, T. (2020). Caracterización de una leche fermentada probiótica endulzada con sirope. Ciencia y Tecnología de los Alimentos, 30(2), 15-
19, ISSN 1816-7721.

Recuperado de:

https://www.revcitecal.iiia.edu .cu/revista/index.php/RCTA/a rticle/view/126

Valdés, M., Guzmán, T., Iñiguez, C., Rodríguez, E., \& Martínez, I. (2020). Empleo de harina de arroz en una leche fermentada probiótica. Ciencia y Tecnología de los Alimentos, 30(1), 38-43, ISSN 1816-7721. Recuperado de: https://www.revcitecal.iiia.edu .cu/revista/index.php/RCTA/a rticle/view/88

Valdés, M., M'Boumba, A., Iglesias, I., Castro, Y., Bejerano, D., Rodríguez, E., ... \& Guzmán, T. (2020). Empleo de pulpas de acerola y guayaba en una leche fermentada probiótica. Ciencia y Tecnología de los Alimentos, 30(2), 10-15, ISSN 1816-7721. Recuperado de: https://www.revcitecal.iiia.edu .cu/revista/index.php/RCTA/a rticle/view/124

Valdés, M., M'Boumba, A., Iglesias, I., Castro, Y., Bejerano, D., Rodríguez, E., ...\& Guzmán, T. (2021). Empleo de pulpas de acerola y mango en una leche fermentada probiótica. Ciencia y Tecnología de los Alimentos, 31(1), 19-23. Recuperado de: https://www.revcitecal.iiia.edu .cu/revista/index.php/RCTA/a rticle/view/218 